\title{
Need-Based Transfers Enhance Resilience to Shocks: An Agent-Based Model of a Maasai Risk-Pooling System
}

\author{
Marco Campennì ${ }^{1}$ - Lee Cronk $^{2}$ - Athena Aktipis ${ }^{1}$
}

Accepted: 1 October 2021 / Published online: 21 October 2021

(c) The Author(s) 2021

\begin{abstract}
Maasai and other Maa-speaking pastoralists in Kenya and Tanzania have a risk-pooling system that they refer to by their word for the umbilical cord (osotua). Gifts from one osotua partner to another are contingent on the recipient's need and do not create any debt. We refer to such gifts as need-based transfers. Maa-speakers also have a system of debt-based transfers (esile) in which gifts must be repaid. We designed an agent-based model to compare the impacts on herd survival of need-based and debt-based transfers on networks of varying topologies and sizes and with different degrees of temporal correlation of shocks felt by the agents. We found that the use of need-based rather than debt-based transfers, greater network modularity, greater network size, and decreased correlation among shocks were associated with increased rates of survival.
\end{abstract}

Keywords Social networks $\cdot$ Risk pooling $\cdot$ Agent-based modelling $\cdot$ Need-based transfers $\cdot$ Small-scale societies $\cdot$ Kenya Tanzania

\section{Introduction}

To pool risk, Maasai and other Maa-speaking pastoralists in Kenya and Tanzania have a livestock-sharing system involving partnerships that they refer to by their word for umbilical cord: osotua (plural isotuatin; Cronk, 2007; Aktipis et al., 2011, 2016; Hao et al., 2015a; Cronk et al., 2019). Many osotua partnerships begin in childhood. Maasai parents encourage their children to form friendships known as isirito (singular: esirit). Isirito share food and exchange small gifts that may become more valuable as they become adults. If both parties agree, they then become isotuatin. Isotuatin rely on each other for help in times of need. For example, if a herder does not have enough livestock to support his family, he may ask one of his osotua partners for enough to bring his family up to the level necessary for survival. Transfers between osotua partners create neither credit nor debt, and Maasai interviewees insist that the concept of payment should never be invoked in an osotua relationship and that there is no expectation of balanced transfers even in the

Marco Campennì

M.Campenni@exeter.ac.uk

1 Department of Psychology, Arizona State University, Tempe, AZ, USA

2 Department of Anthropology, Rutgers University, New Brunswick, NJ, USA long run. Transfers occur only in response to requests, and requests must arise from genuine need and must be limited to the amount actually needed. Osotua relationships are imbued with respect, restraint, and a sense of great responsibility (Cronk, 2007; Cronk et al., 2019).

People often try to establish osotua partnerships with people in different ecological zones so that their risk profiles are complementary (see also Gulliver, 1955; Dixit et al., 2013). For example, highland areas are relatively well-watered, but they harbor more pathogens than do dryer lowland areas. Unfortunately, droughts and other calamities sometimes affect large areas. As a result, an individual herder may have trouble finding any isotuatin who are able to help. Such a situation of highly correlated shocks occurred among the Maasai during a nineteen-year period in the late 19th and early twentieth centuries that they refer to as Emutai, meaning, roughly, "the wiping out" (Waller, 1988). Emutai was caused by a combination of drought and epidemics among both humans and their livestock and led to a breakdown of social relations and civil war among Maasai sections.

In addition to osotua, Maasai and other Maa-speakers have several other rules that govern property transfers in a variety of specific circumstances. Among Maasai living in Ngorongoro, a clan-based system of aid through livestock transfers called engelata (i.e., "choice," Mol, 1996:79) has been documented (Potkanski, 2000). Several varieties of engelata exist. Engelata enkaputee (i.e., of the affines) is 
a way to raise bridewealth. Engelata ewoloto is a way for a clan to help a destitute family. The Maa-speaking Samburu of northern Kenya refer to the lending of a cow's milk to another household as keruu and the lending of the cow itself as ketaaro, while aitogaroo refers to the loan of a bull for breeding purposes, and ketapashaki refers to the immediate exchange of two animals, e.g., a steer for a cow. Livestock may also be transferred in the form of bridewealth and as compensation for crimes (Perlov, 1987; Potkanski, 2000). One type of livestock transfer that contrasts sharply with osotua is esile, which translates simply as "debt." In esile, unlike in osotua, credit and repayment of debts are the essence of the relationship: both parties expect that the debt will be repaid. "An esile is like a bank" is a common expression among the Samburu that captures this idea (Perlov, 1987:186). As with bank loans, esile transfers, unlike ketapashaki, involve a delay between the loan and its repayment. Common reasons for requests of esile are the desire to make some cash by selling livestock or because one needs a specific type of animal that one does not already have for a particular ceremony.

Because many other pastoralist societies also have sharing rules equivalent or similar to osotua and esile (Almagor, 1978; Bollig, 1998, 2006; Dyson-Hudson, 1966; Flannery et al., 1989; Gulliver, 1955; Iyer, 2016; Moritz, 2016; Moritz et al., 2011), we henceforth follow Aktipis et al. (2016) and Cronk et al. (2019) in using a more general, less Maasaispecific set of terms, referring to osotua as "need-based transfers" and esile as "debt-based transfers."

Four previous efforts have been made to use computer simulations to model aspects of Maasai-style risk-pooling networks. In all of these simulations, agents begin with an amount of livestock that is slightly above a fixed threshold for survival. Agents who drop below that threshold for a predetermined number of rounds (e.g., two) drop out of the simulation and are considered to have not survived, although in the real world such impoverished herders might instead supplement herding with other food sources such as hunting, gathering, and farming. During each round, herds grow, but they may also experience losses that simulate the impacts of droughts, diseases and thefts. Agents are also programmed with decision-making rules for how and when to transfer cattle that vary from simulation to simulation. Aktipis et al. (2011) created an agent-based model in which agents endowed with herds that grew and experienced occasional negative shocks were paired with each other in dyads. The model included four types of agents: (1) those that followed a need-based transfer rule (i.e., ask when in need, give if able); (2) those that never transferred anything; (3) those that asked their partner for cattle at a rate equivalent to the average asking rate among agents following a need-based asking rule and who requested a number of cattle equivalent to the mean amount given by agents in the need-based transfer runs; and (4) those that, when asked for help, gave a proportion of their cattle that was equivalent to the average mean proportion given in the need-based transfer runs. Agents following the full need-based transfer rule survived longer than all other types of agents. Aktipis et al. (2016) also placed agents in dyads, finding that agents following need-based transfer rules outperformed those following debt-based transfer rules.

Different network features and correlation of disasters can affect the outcomes for agents sharing on networks using need-based transfer rules. Hao et al. (2015a, b) found that agents arranged in a network and following a need-based transfer rule survived longer when those in need asked their wealthiest partner rather than a partner chosen at random and that greater network connectedness improved survival. Hao et al. $(2015$, b) found that temporal correlation of disasters decreased the survival of need-based agents while spatial correlation of disasters improved their survival. The authors attributed the improvement in survival when disasters are spatially correlated to a phenomenon they label "disaster masking": A given disaster is likely to kill only a small, highly localized group of agents, leaving the rest to thrive while also clearing agents out of a location where additional disasters are likely to strike in the future.

In the present paper, we build upon this previous work to address several questions. Like Hao et al. (2015a) and Hao et al. (2015b), we model agents on a network, but we also vary both network size and network topology. Like Hao et al. (2015b), we examine temporally correlated disasters: It is clearly better if agents do not experience shocks simultaneously, but where is the threshold at which temporal correlation spells doom, and does that threshold change depending on the settings of the other parameters? Finally, like Aktipis et al. (2016) we compare the survival of need-based transfer agents with that of debt-based transfer agents, but here we vary the sizes and topologies of social networks as well as the temporal correlations of disasters. Giving to those in need, often with no expectation of repayment, is found not only in the Maasai osotua system but also in many other risk-pooling systems documented ethnographically in small-scale societies around the world, which indicates that it may be adaptive in a wide variety of ecological and economic circumstances (Bird et al., 2002; Bollig, 1998, 2006; Cashdan, 1985; Cronk et al., 2019; Fafchamps, 2011; Fafchamps \& Lund, 2003; Gulliver, 1955; Iyer, 2016; Smith et al., 2019; Wiessner, 1982). Here we investigate the performance of need-based transfer and debt-based transfer strategies on three different network topologies: regular networks, small-world networks and preferential attachment networks. 
We tested the following hypotheses:

1. Agents following a need-based transfers rule will survive longer than agents following a debt-based transfer rule.

2. Agents in larger networks will survive longer than agents in smaller networks.

3. Agents in more modular networks will survive longer than agents in less modular networks.

4. Agents that experience shocks that are less correlated temporally with shocks experienced by other agents in their networks will survive longer than agents that experience shocks that are more correlated temporally with shocks experienced by other agents in their networks.

\section{Methods}

We used NetLogo (Wilensky, 1999) to model a population of agents in networks with varying topologies (see Fig. 1). We included regular networks, small-world networks, and preferential attachment networks in our simulations. In regular networks, all individuals are connected to the same number of individuals within the network. In a small-world network, there are a number of connections that span across the network, leading to the phenomenon seen in the real world in which strangers can be linked through a small chain of acquaintances. We also included preferential attachment networks. Using the Barabási and Albert model (Barabási \& Albert, 1999), we generated random scale-free networks using a preferential attachment mechanism, which preferentially adds connections to nodes that are already well connected (see glossary).

As in Aktipis et al. $(2011,2016)$ each node/agent in the network represents a household/family of approximately six individuals, and each link represents a connection to another household. The model is parameterized based on the ecology of East Africa, and the rules that agents use are based on the osotua (need-based transfer) and esile (debt-based transfer) rules used by Maasai.

During each time step (representing a year), households experience herd growth and potential shocks and losses. They also have an opportunity to ask for and give help to other households (see Fig. S1). An agent's initial herd of 70 grows or shrinks during each time step of the simulation at a rate normally distributed around a mean of $3.4 \%$. The minimum viable herd size was set at 64 , based on estimates of a family's caloric needs and productivity in the dry season. The maximum cattle herd size allowed in the model is 600 , which represents a realistic approximation of the maximum cattle herd size for an average-sized household. During each time step there is also a chance of a loss (e.g., through a drought or a disease spreading in the herd). All of these parameters, including the likelihood and severity of these losses, were estimated based on Dahl and Hjort's study of the actual dynamics of East African cattle herds (1976).

The model was run for 100-time steps. Across models we vary the probability, $p_{\text {shocks }}$, that a loss is correlated (i.e., it affects all households at the same time) from 0 to 1 with steps of 0.1 .

We simulated both need-based and debt-based livestock transfers between households as follows:

Need-based transfer rule: Need-based transfer agents monitor their livestock holdings. If they drop below the critical threshold for livestock holdings, they ask their wealthiest partner for help (i.e., agents used a selective asking rule rather than a random asking rule as in Hao et al., 2015a); if they are asked for help and can afford to provide it without putting themselves below the critical threshold for survival, they do so. The pseudo-code (i.e., a description in natural language rather than computer code) for the need-based transfer rules were implemented as follows (as in Aktipis et al., 2016, Aktipis et al., 2011, and Hao et al., 2015a):

1. Need-based asking rule: Individuals ask their partners for livestock only if their current holdings are below the asking threshold (i.e., the minimum herd size of 64).

2. Need-based giving rule: Individuals give what is asked, but not so much as to put their herds below the giving threshold (also the minimum herd size of 64).

Debt-based transfer rule: Debt-based transfer agents also ask their wealthiest partner for help when they are in need, but otherwise, they differ from need-based transfer agents. Debt-based agents transfer cattle only if they are asked by a partner who is in good standing. These debtbased agents keep track of the amounts they owe to and were owed by the other agents in their networks. Recipients of loans repay these loans as soon as they have enough livestock to do so without going below the sustainability threshold of 64 units. If five rounds go by after a transfer without repayment, then the agent who gave the loan considers the 'defaulting' partner to no longer be in good standing and therefore does not provide a loan to that agent in the future. The pseudo-code for this rule is as follows:

\section{Debt-based payback rule:}

If livestock have been previously transferred from the partner to the actor and the actor has enough to pay back without going below the sustainability threshold (resource min), the actor pays livestock back to his partner according to the actor's repayment probability 


$$
N=30
$$
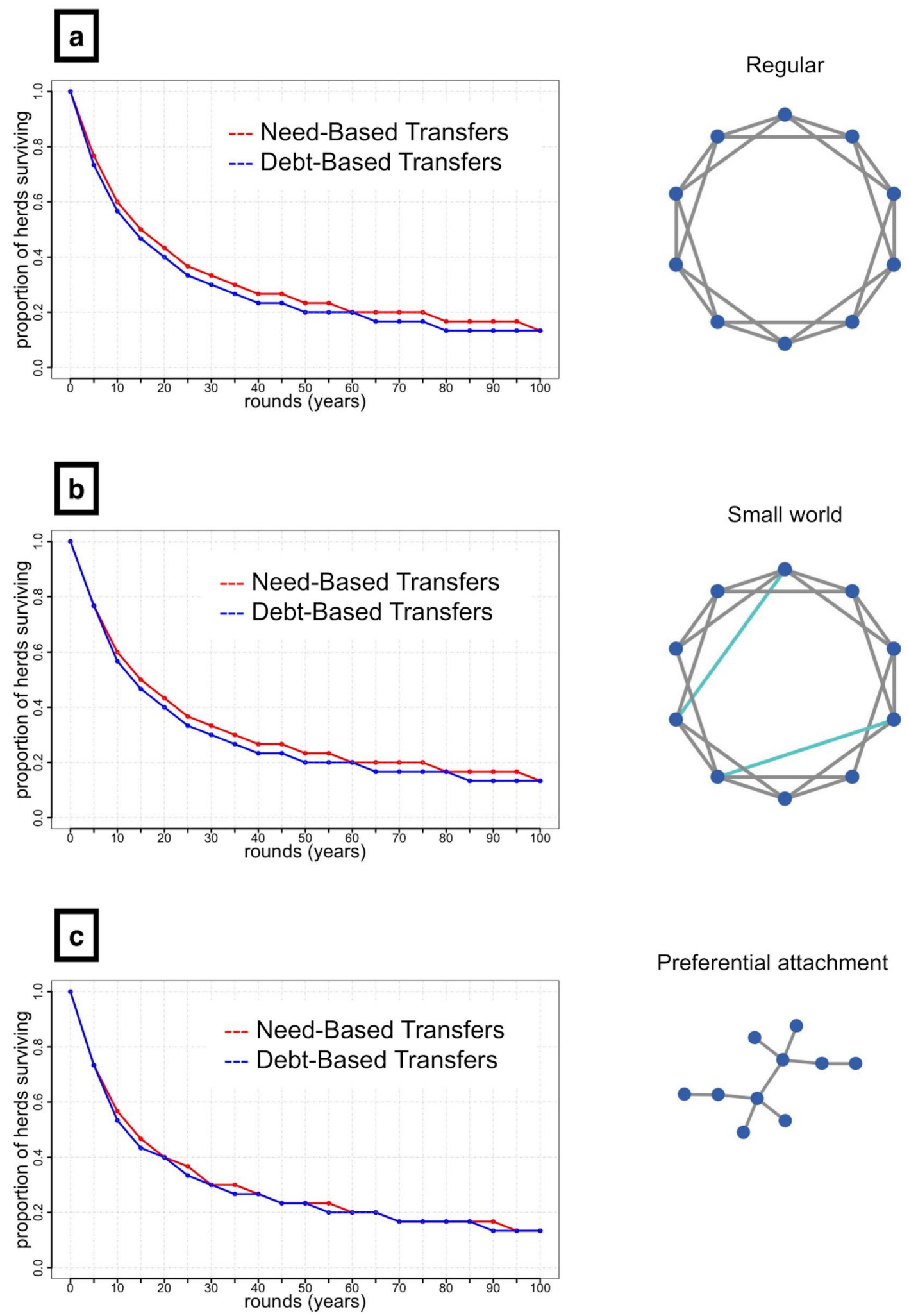

Preferential attachment

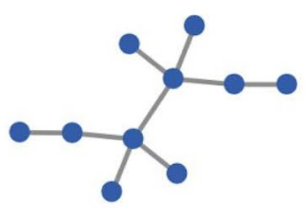


4Fig. 1 Proportion of herds surviving (y-axis) as a function of time (x-axis), for three network topologies (regular, small-world and preferential attachment), $\mathrm{N}=30$, when there is no correlation of shocks. Need-based agents significantly outperform debt-based agents across all network topologies (see Table 3 and Tables S1-S5)

\section{Debt-based partner credit check rule:}

Checks whether a partner is in good standing, which includes not having exceeded tolerated delay or credit size (when applicable)

\section{Debt-based asking rule:}

As with the need-based transfer asking rule, individuals ask their partners for livestock if their current herd size is below the sustainability threshold of 64.

\section{Debt-based giving rule:}

Response to partner. If a request is made, actors give if two conditions are met:

1. If no debt remains from a previous request and the partner is in good standing (meaning that previous debt had not existed for longer than tolerated delay)

2. The amount transferred cannot exceed the credit size extended to the partner

We varied four key parameters while keeping all other aspects of the simulations constant:

(1) Network topology: regular, small world, and preferential attachment.

(2) Network size: values of 30, 40, 50, 60, 70, 80, 90, and 100 .

(3) Correlation of shocks: probabilities of $0,0.1,0.2,0.3$, $0.4,0.5,0.6,0.7,0.8,0.9$ and 1 .

(4) Sharing rule: need-based transfers vs. debt-based transfers.

This yielded a total of 528 unique combinations of parameters for our simulations. For each of these 528 combinations of parameters, we ran the model 10,000 times.

\section{Results}

We varied four parameters: network topology, network size, sharing rule, and correlation of shocks. For each combination of these parameters, we tested the performance of two resource transfers rules, i.e., a need-based strategy and a debt-based strategy. Unless otherwise specified, all results refer to small-world networks, which are the default type of network used for these kinds of studies. Also, in the graphical presentation of our results, we often show only a subset of the full data because it is impossible to show the full dimensionality of the data given that we varied four parameters (network topology, network size, correlation of shocks, and the sharing rule).

In a separate set of simulations, we varied tolerated delay, sampling values from one year to 50 years of tolerated delay. The tolerated delay parameter is relevant only to debt-based transfer agents; it specifies how long they will wait to get paid back before refusing to interact with that partner again. Results show that the impact of variations in the tolerated delay is minimal compared to the impacts of the other parameters we varied. We, therefore, set tolerated delay at five years, which is in line with our previous publication (Aktipis et al., 2016).

Here we report the survival of agents (i.e., households), as a proxy for the ecological viability of these strategies. However, we have also run evolutionary versions of the model (i.e., versions in which agents can reproduce), and we present preliminary results from those simulations in the Supplementary Information (Fig. S2).

We used two different measures to evaluate the performance of the two sharing strategies. First, we calculated the ratio of runs resulting in the survival of at least one agent from the initial population of $\mathrm{N}$ agents at the end of the runs (time step 100). This is a measure of the effectiveness of adopting a specific sharing strategy given a set of conditions (i.e., network topology, group size, and correlation of shocks). Given a specific combination of parameters, this ratio is expressed as the number of runs ending with at least one surviving agent over the total number of runs (i.e., 10,000 runs). Second, we examined the proportion of herds surviving at the end of each run (time step 100) calculated for each combination of the parameters as the median value from all 10,000 runs over the initial N. Finally, because the simulations' results are not normally distributed and because our sample size is so large that p-values alone may not be sufficient to draw strong conclusions from our results, we have included two additional analyses. One of these is a Mann Whitney U test (Hollander \& Wolfe, 1973) (the results of which are presented throughout this section and in Supplementary Information), and the other is a report simply of all of the conditions in which the survival advantage of the need-based strategy is greater than 5\% over the debt-based strategy (presented at the end of the results section).

We used the Mann-Whitney U statistic to test whether the need-based strategy has stochastic dominance compared with the debt-based strategy. This allows us to verify the null hypothesis that results from both strategies follow the same distribution, quantifying the probability that a randomly 
selected datum from a first set of results (e.g., the needbased strategy results) is higher or lower than another datum randomly selected from a different set of results (e.g., the debt-based strategy results). Some differences between the performance of the strategies in the model are small, and the $\rho$ statistic allows us to evaluate whether the effect size is meaningful.

The $\rho$ statistic has values ranging from zero to one. A value of 0.5 means that the two sets of data overlap. The closer it is to 1 , the greater the stochastic dominance of the first set of results; the closer it is to 0 , the greater the stochastic dominance of the second set of results. To define the statistical significance of the values obtained by running the Mann-Whitney U test, we have combined the $\rho$ statistic and $\mathrm{p}$-values to derive a cut-off. If the value of the $\rho$ statistic is between 0.49 and 0.51 , we considered this a confirmation of the null hypothesis that two distributions of data overlap and therefore, there is no difference in the performance of the need-based and debt-based strategies.

\section{Network Topology}

Regardless of network topology, agents using the needbased rule outperform agents using a debt-based rule when shocks are uncorrelated. In Fig. 1 we show the proportion of herds surviving over time as the simulations progress. Table 1 shows the proportion of runs ending with at least one surviving agent (see also Table 3 and Tables S1-S2 in Supplementary Information, which provides a measure of the effect size calculated as $\rho$ from the Mann Whitney $U$ test). If we compare survival across different network topologies, we can see that more agents in preferential attachment networks survive, on average, than do agents in regular and small-world networks when $\mathrm{N}<50$ (see Table 1). The improvement in survival for agents in modular preferential attachment networks (compared to other networks) is significant for agents following either transfer rule (see Fig. 1a, c and the first row of Table 1). In preferential attachment networks, sub-groups of nodes are highly interconnected within groups but loosely connected outside them, which can help to limit the impact of shocks on the network as a whole. As a result, the difference in performance between need-based and debt-based systems is smaller for the preferential attachment network structure compared to other network structures (this difference in performance is also limited to no correlation of shocks and networks of small size, i.e., $\mathrm{N}<60$ when the correlation is 0.1 and $\mathrm{N}<40$ when the correlation is 0.2 . See Table S2 in Supplementary Information for details about the effect size as $\rho$ from the Mann Whitney U test).

Although, on average, the proportion of runs ending with at least one surviving agent is the same for both strategies when $\mathrm{N}>30$ (see rows 2 and 3 in Table 1), Mann Whitney $\mathrm{U}$ test effect size $\rho$ shows that, when shocks are uncorrelated, the need-based rule stochastically dominates the debt-based rule for all values of $\mathrm{N}$ (Mann Whitney $\mathrm{U}$ test $\rho>0.51$ and p-value $<0.05$ in all cases, except for preferential attachment networks with $\mathrm{N}=80$, where $\rho=0.508$, but $\mathrm{p}$-value $<0.05$; see first column of Tables 3, S1-S2, and first column of Tables S3-S5).

\section{Network Size}

In order to see how the size of a network affects the ability of agents to survive, we considered a small-world network, $\mathrm{p}_{\text {shocks }}=0.1,0.5$, and 1 , and varied network size (see Fig. S3a-f and Table 2). Particularly for networks with $N<100$ and low correlation of shocks $\left(\mathrm{p}_{\text {shocks }}=0.1\right)$, agents following a need-based transfer rule significantly outperformed those following a debt-based transfer rule (Mann Whitney $\mathrm{U}$ test derived $\rho=0.553$ when $\mathrm{N}=30, \rho=0.546$

Table 1 The proportion of runs ending with at least one surviving agent at time round $=100$, varying the size of the networks $(\mathrm{N}=30,50,100)$. This table shows a subset of the results obtained by systematically varying N. NBT $=$ need-based transfer rule, DBT $=$ debt-based transfer rule

\begin{tabular}{|c|l|l|l|l|l|l|}
\hline$N$ & $\begin{array}{l}\text { Regular } \\
\text { NBT }\end{array}$ & $\begin{array}{l}\text { Regular } \\
\text { DBT }\end{array}$ & $\begin{array}{l}\text { Small World } \\
\text { NBT }\end{array}$ & $\begin{array}{l}\text { Small World } \\
\text { DBT }\end{array}$ & $\begin{array}{l}\text { Preferential } \\
\text { NBT }\end{array}$ & $\begin{array}{l}\text { Preferential } \\
\text { DBT }\end{array}$ \\
\hline 30 & 0.983 & 0.973 & 0.985 & 0.972 & 0.99 & 0.985 \\
\hline 50 & 0.999 & 0.999 & 0.999 & 0.999 & 1 & 0.999 \\
\hline 100 & 1 & 1 & 1 & 1 & 1 & 1 \\
\hline
\end{tabular}


<smiles>c1ccc(C23CC4CC(CC(C4)C2)C3)cc1</smiles>

a

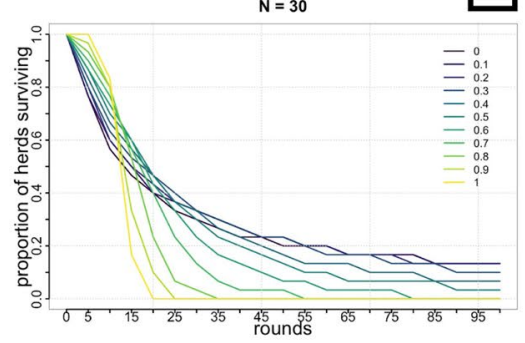

回

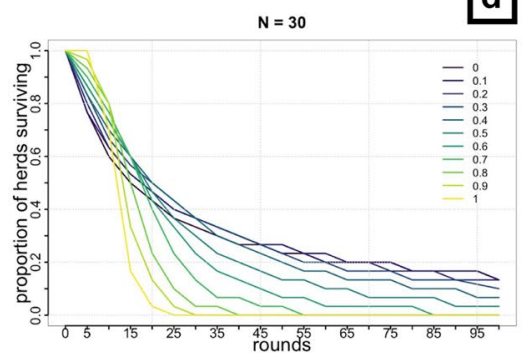

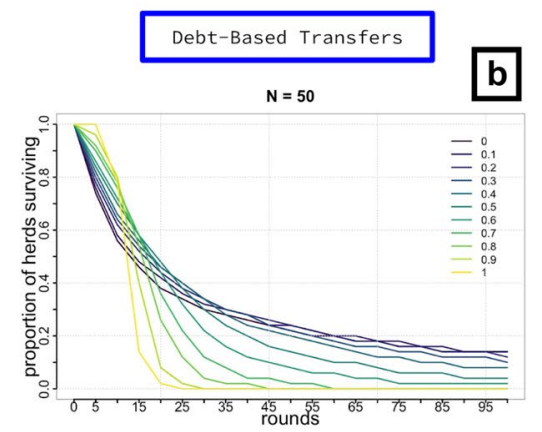

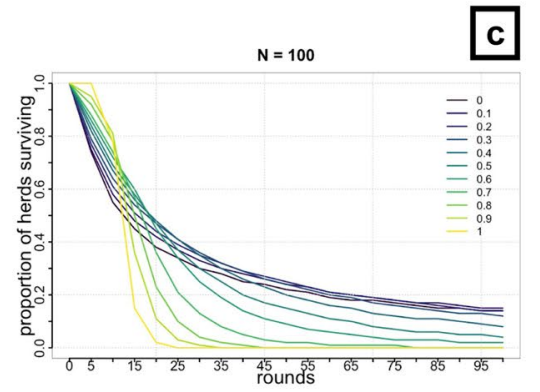

Need-Based Transfers

e

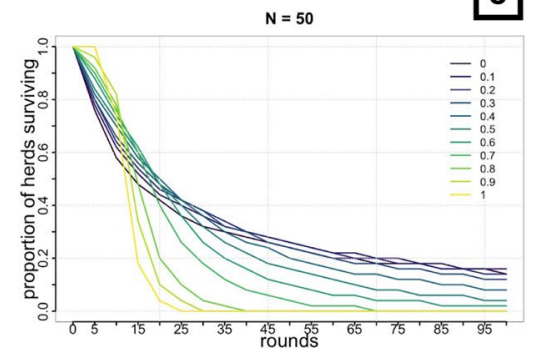

f

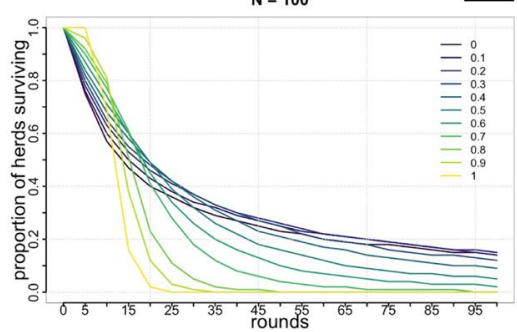

Fig. 2 Proportion of herds surviving for agents using debt-based (a-c) and need-based (d-e) strategies in small world networks for three different network sizes $(\mathrm{N}=30,50,100)$. The figure shows the proportion of herds surviving (y-axis) as a function of the correlation of shocks.

when $\mathrm{N}=50$, and $\rho=0.519$ when $\mathrm{N}=100$; see Table 3 , and Table S3 for details). As network size increases, survival rates increase for agents following both rules, though the need-based rule continues to perform better than the debt-based rule as long as the correlation of shocks is low or intermediate (see Table 2a and the second column of Table 3 for Mann Whitney U test $\rho$ values). As the correlation of shocks increases, the survival of both strategies diminishes, and the advantage of the need-based strategy over the debt-based strategy becomes smaller and eventually not significant (see Table $2 \mathrm{~b}$ and sixth column of Tables 3 and S3).

When shocks are completely correlated $\left(\mathrm{p}_{\text {shocks }}=1\right)$, the Mann-Whitney U test reveals that the performances of the need-based and debt-based strategies are not different (see Table $2 \mathrm{c}$ and last column of Tables 3 and S3).

\section{Correlation of Shocks}

If everyone in a social network experiences a severe shock simultaneously, no one may be in a position to help anyone else. To investigate the effects of synchronicity of shocks, we investigated three network sizes $(\mathrm{N}=30,50,100)$ and varied the correlation of shocks
Need-based strategies outperform debt-based strategies for networks of size $\mathrm{N}=30,50,100$ and correlation of shocks $<0.5$ when $\mathrm{N}=30,50$ and correlation of shocks $<0.3$ when $\mathrm{N}=100$ (Mann Whitney U test $\rho$ $>0.51$; see Tables 2 and 3 for details)

$\left(\mathrm{p}_{\text {shocks }}=[0,1]\right)$ from a scenario in which all shocks are uncorrelated (i.e., an idiosyncratic scenario) to a scenario in which all shocks are completely correlated (i.e., a systemic scenario), affecting all agents at the same time (see Fig. 2). This probability, $\mathrm{p}_{\text {shocks }}$, is the likelihood that a shock will affect all agents in a network at the same time. When $p_{\text {shocks }}=0$, a shock is likely to affect just a single agent in the network at time t. At the other extreme, when $\mathrm{p}_{\text {shocks }}=1$, all agents from the same network are affected by the same shock at the same time. We also considered intermediate scenarios by varying the probability of correlated shocks from 0 to 1 by steps of 0.1 . For low levels of correlated shocks, need-based agents outperform debt-based agents (Mann Whitney $U$ test $\rho$ values $>0.51$ for all values of $\mathrm{N}$, when $\mathrm{p}_{\text {shocks }}<0.3$; $\rho$ values $>0.51$ for $\mathrm{N}<80$, when $\mathrm{p}_{\text {shocks }}<0.5 ; \rho$ values $>0.51$ for $\mathrm{N}<60$, when $\mathrm{p}_{\text {shocks }}<0.7$; see Tables 3 and S3 for details). As the probability of correlated shocks increases, survival of agents decreases, and both strategies perform equally poorly when the probability of correlated shocks is higher than 0.7 (see Table 2 showing the proportion of runs ending with at least one surviving agent considering $\mathrm{p}_{\text {shocks }}=0.1,0.5,1$ and Table 3 showing the effect 
Table 2 The proportion of runs resulting in the survival of at least one agent, varying the size of the networks $(\mathrm{N}=30,50,100)$ and the correlation of shocks $\left(\mathrm{p}_{\text {shocks }}=0.1,0.5,1\right.$, in panels $\mathrm{a}, \mathrm{b}, \mathrm{c}$, respec- tively). This table shows the results obtained by systematically varying $\mathrm{N}$ and $\mathrm{p}_{\text {shocks }}$. NBT = need-based transfer rule, DBT $=$ debt-based transfer rule

\begin{tabular}{|c|c|c|c|c|c|c|c|}
\hline & $N$ & $\begin{array}{l}\text { Regular } \\
N B T\end{array}$ & $\begin{array}{l}\text { Regular } \\
D B T\end{array}$ & $\begin{array}{l}\text { Small World } \\
\text { NBT }\end{array}$ & $\begin{array}{l}\text { Small World } \\
\text { DBT }\end{array}$ & $\begin{array}{l}\text { Preferential } \\
N B T\end{array}$ & $\begin{array}{l}\text { Preferential } \\
D B T\end{array}$ \\
\hline \multirow{4}{*}{0.1} & 30 & 0.877 & 0.862 & 0.876 & 0.861 & 0.896 & 0.889 \\
\hline & 50 & 0.909 & 0.908 & 0.917 & 0.904 & 0.933 & 0.931 \\
\hline & 100 & 0.936 & 0.936 & 0.942 & 0.937 & 0.945 & 0.942 \\
\hline & 30 & 0.549 & 0.522 & 0.537 & 0.533 & 0.578 & 0.577 \\
\hline \multirow[t]{3}{*}{0.5} & 50 & 0.583 & 0.57 & 0.583 & 0.574 & 0.615 & 0.623 \\
\hline & 100 & 0.636 & 0.622 & 0.626 & 0.622 & 0.655 & 0.639 \\
\hline & 30 & 0.241 & 0.239 & 0.24 & 0.24 & 0.25 & 0.256 \\
\hline \multirow[t]{2}{*}{1} & 50 & 0.265 & 0.251 & 0.261 & 0.253 & 0.266 & 0.274 \\
\hline & 100 & 0.284 & 0.273 & 0.276 & 0.28 & 0.292 & 0.287 \\
\hline
\end{tabular}

size $\rho$ from the Mann Whitney $\mathrm{U}$ test: when $\mathrm{p}_{\text {shocks }}>0.7$ neither strategy stochastically dominates $\rho<0.51$, except for the specific combination of parameters $\mathrm{N}=60$ and $\mathrm{p}_{\text {shocks }}=0.8$ ).

\section{Conditions in which the Survival Advantage is Greater than $\mathbf{5 \%}$ for Need-Based vs. Debt-Based Strategies}

In order to identify the combinations of conditions in which the difference between need-based and debt-based strategies is largest, we considered all the combinations of parameters in which need-based agents had at least a 5\% survival advantage over the debt-based agents. We calculated the difference of median herds surviving at the end of the simulations $(t=100)$ (see Eq. 1).

advantage $_{N B T \text { over } D B T}=\frac{M_{i, j}^{N B T}-M_{i, j}^{D B T}}{M_{i, j}^{D B T}}$

where $M_{i, j}^{N B T}$ is the matrix of the median herds surviving of need-based strategy networks at the end of the simulations in which columns stay for different values of $\mathrm{p}_{\text {shocks }}$ (from 0 to 1 by 0.1 ) and rows represent network size (from 30 to 100 by 10$)$;

$M_{i, j}^{D B T}$ is the matrix of the median herds surviving of debtbased strategy networks at the end of the simulations in which columns stay for different values of $\mathrm{p}_{\text {shocks }}$ (from 0 to 1 by 0.1 ) and rows represent network size (from 30 to 100 by 10); and $M_{i, j}^{D B T}>0$.

\section{Small World Networks}

We found that, in small world networks, when the probability of concurrent shocks is low $\left(\mathrm{p}_{\text {shocks }}<=0.2\right)$, the need-based rule outperforms the debt-based rule by $5 \%$ or more in $50 \%$ of cases. When the probability of concurrent shocks is intermediate $\left(0.3<=\mathrm{p}_{\text {shocks }}<0.5\right)$ need-based agents have an advantage of $5 \%$ or more in $37.5 \%$ of cases. When $\mathrm{p}_{\text {shocks }}=0.5$ the advantage of $5 \%$ or more is in $25 \%$ of cases and when $\mathrm{p}_{\text {shocks }}=0.6$ it is in $12.5 \%$ of cases. Finally, when $p_{\text {shocks }}>0.6$, need-based rule systems never outperform debt-based rule systems (see Table S6). The general pattern of results fits our earlier findings that for lower probabilities of correlated shocks, needbased transfer agents significantly outperform debt-based 
Table 3 Small World Networks. This table shows the effect size $\rho$ calculated from the Mann-Whitney U test normalized by the product of sizes of groups (see Tables S3-S5 in Supplementary for associated $\mathrm{p}$-values). Columns represent different values for $\mathrm{p}_{\text {shocks }}$ while rows represent different network sizes. We can arbitrarily set two thresholds at 0.49 and 0.51 , assuming that all values in between are close enough to 0.5 to be considered "as if 0.5 " and therefore showing that there is no stochastic dominance of either group. This arbitrary cutoff is confirmed by p-values (see Tables S3-S5 in Supplementary). Values outside that interval $(0.49-0.51)$ are presented in red. All values shown in red are for cases in which there is stochastic dominance of need-based transfers over debt-based transfers; in no cases did debt-based transfers have stochastic dominance over need-based transfers

Small World Networks: $\rho$ values from Mann Whitney $U$ test

$\begin{array}{rccccccccccc} & \mathbf{0} & \mathbf{0 . 1} & \mathbf{0 . 2} & \mathbf{0 . 3} & \mathbf{0 . 4} & \mathbf{0 . 5} & \mathbf{0 . 6} & \mathbf{0 . 7} & \mathbf{0 . 8} & \mathbf{0 . 9} & \mathbf{1} \\ 30 & 0.592 & 0.553 & 0.533 & 0.519 & 0.526 & 0.509 & 0.512 & 0.505 & 0.504 & 0.506 & 0.5 \\ 40 & 0.577 & 0.548 & 0.527 & 0.519 & 0.518 & 0.516 & 0.512 & 0.509 & 0.505 & 0.504 & 0.504 \\ 50 & 0.567 & 0.546 & 0.528 & 0.521 & 0.51 & 0.511 & 0.514 & 0.514 & 0.499 & 0.502 & 0.504 \\ 60 & 0.562 & 0.536 & 0.524 & 0.513 & 0.515 & 0.51 & 0.501 & 0.499 & 0.511 & 0.501 & 0.503 \\ 70 & 0.567 & 0.528 & 0.524 & 0.518 & 0.518 & 0.5 & 0.502 & 0.507 & 0.502 & 0.501 & 0.499 \\ 80 & 0.554 & 0.523 & 0.525 & 0.509 & 0.506 & 0.513 & 0.506 & 0.501 & 0.507 & 0.496 & 0.502 \\ 90 & 0.551 & 0.526 & 0.515 & 0.509 & 0.512 & 0.505 & 0.501 & 0.505 & 0.503 & 0.502 & 0.502 \\ 100 & 0.555 & 0.519 & 0.517 & 0.506 & 0.509 & 0.511 & 0.511 & 0.512 & 0.503 & 0.495 & 0.499\end{array}$

transfer agents (see Tables 3 and S3). There was only one combination of parameters where the debt-based strategy outperformed the need-based strategy in small-world networks: 70 agents at an intermediate shock correlation $\left(\mathrm{p}_{\text {shocks }}=0.5\right)$. However, this combination of parameters had no effect size and was not statistically significant in our earlier analyses (see Tables 3 and S3).

\section{Regular Networks}

In regular networks, when the probability of shocks is low ( $\mathrm{p}_{\text {shocks }}<=0.2$ ), the need-based rule outperforms the debt-based rule by $5 \%$ or more in $58.3 \%$ of cases. When the probability of concurrent shocks is intermediate $(0.3<=$ pshocks $<0.5)$ need-based agents have an advantage of $5 \%$ or more in $43.75 \%$ of cases. When $\mathrm{p}_{\text {shocks }}=0.5$, the advantage of $5 \%$ or more appears in $25 \%$ of cases; when $\mathrm{p}_{\text {shocks }}=0.6$ they have this advantage in $12.5 \%$ of cases. Finally, when $\mathrm{p}_{\text {shocks }}>0.6$, need-based rule systems never outperform debt-based rule systems (see Table S7). There were no combinations of parameters in which the debt-based agents outperformed the need-based agents in regular networks.

\section{Preferential Attachment Networks}

Finally, in preferential attachment networks, when the probability of shocks is low ( $\mathrm{p}_{\text {shocks }}<=0.2$ ), the need-based rule outperforms the debt-based rule by $5 \%$ or more in $16.67 \%$ of cases. When the probability of concurrent shocks is intermediate $\left(0.3<=\mathrm{p}_{\text {shocks }}<0.5\right)$ need-based agents have an advantage of $5 \%$ or more in $25 \%$ of cases. When $\mathrm{p}_{\text {shocks }}=0.5$, needbased agents have an advantage of $5 \%$ or more in $12.5 \%$ of cases, and when $\mathrm{p}_{\text {shocks }}=0.6$ they have an advantage in $25 \%$ of cases. Finally, when $\mathrm{p}_{\text {shocks }}>0.6$, need-based rule systems never outperform debt-based rule systems (see Table S8). Debt-based agents did not outperform the need-based agents under any parameter combinations in preferential attachment networks.

\section{Discussion}

We created an agent-based model to investigate how the network topology, the size of the network, and the correlation of shocks affected the viability of need-based and debt-based transfer strategies. We found that agents survived longer in modular preferential attachment networks than in regular and small-world networks. We also found that larger network sizes increased survival, that higher correlations among shocks decreased survival, and that agents following a need-based transfer rule were generally more likely to survive than those following a debtbased transfer strategy. This advantage of need-based over debt-based strategies was especially clear in modular and small networks when correlations among shocks were low. 
Although there were conditions in which the need-based and debt-based strategies did equally well (e.g., when shocks were highly correlated), there were no conditions in which the debt-based strategy outperformed the needbased strategy.

\section{Limitations of the Success of Need-Based Over Debt-Based Transfers}

Transfers to those in need, often with no expectation of repayment, are common in human risk-pooling systems such as central-place food sharing among foragers and the osotua system of the Maasai (Bird et al., 2002; Cronk et al., 2019). This suggests that need-based transfer systems may have an adaptive advantage over other transfer rules (Cronk $\&$ Aktipis, 2021). As we have seen, the need-based sharing rule outperforms the debt-based sharing rule when networks are modular and small and when shocks are not highly correlated. However, the advantage of need-based strategies over debt-based strategies disappears when shocks are highly correlated, and both strategies do equally poorly.

\section{Does the Modularity of Networks Increase their Resilience?}

Networks that are more modular tend to be more resilient to perturbations (Ash \& Newth, 2007; Bettencourt et al., 2007; Bullmore \& Sporns, 2012; Eriksen et al., 2003; Garas et al., 2008; Guimera et al., 2005). Modular networks are typically more resilient against cascading failures. Self-contained or modular components can help to isolate shocks and prevent the cascade of negative effects through the network (Ash \& Newth, 2007). However, scale-free networks can sometimes be more vulnerable to targeted attacks than other networks if those attacks are concentrated on more connected nodes (Lenzu \& Tedeschi, 2012).

Generally speaking, however, networks that are less modular (e.g., regular networks and small-world networks) are more vulnerable to the effects of shocks. Future work should investigate which characteristics of preferential attachment networks are driving this effect-in particular, whether the modularity of the network is the key factor or whether other network factors drive the effect. In our simulations, preferential attachment networks also have a higher average degree (i.e., the number of connections or edges that nodes have to other nodes) and higher average Eigenvector centrality (i.e., a measure of the influence of a node in a network).

How relevant this finding is to the social networks of Maasai and other pastoralists is unclear. Although we do know that some human social networks are modular (e.g., Newman, 2006), we do not know the structure of real-world risk-pooling networks specifically. Information about such networks' parameters could be used as the basis for future efforts to create computer simulations of such networks.

\section{Correlated Disasters Strain the Resilience of Resource Transfer Systems}

Our finding that correlated disasters lead to much lower survival than uncorrelated ones replicates an earlier model of osotua networks (Hao et al., 2015b) and is similar to that of a model of shocks in financial systems (Steinbacher et al., 2016). The authors of that paper argue that the detrimental impact of systemic shocks comes from the fact that the shocks themselves reduce the wealth of each node of the network in the system at the same time, which makes them more vulnerable to shocks in subsequent periods. Hence, large shocks are likely to be followed by cascading defaults, thereby destabilizing the whole system.

When shocks are highly correlated, the result may be that no transfers occur because no one can afford to help anyone else. Such situations have been known to arise during severe famines, as during the Emutai period among the Maasai mentioned in the introduction. Similarly, in the 1960 s, the Ik, a group of former hunter-gatherers in northeastern Uganda, suffered from such a severe famine that an ethnographer present at the time noted a marked lack of sharing, even among close kin (Turnbull, 1972). Although that ethnographer interpreted the lack of sharing as an aspect of their cultural traditions (rather than as a response to their extremely dire circumstances), subsequent work has shown that their culture actually celebrates and encourages sharing and that Ik routinely share with those in need when they are able to do so (Townsend et al., 2020). These findings from the field are consistent with the model results showing that correlated shocks present a unique challenge to overall survival in a network, even when generous sharing rules are in place.

The superiority of need-based transfers over debt-based transfers in these models can be attributed to the simple fact that debt-based agents sever ties with other agents who, particularly in a volatile environment, might actually be helpful to them at some point in the future. Need-based agents, in contrast, do not expect to be repaid and so maintain all their partnerships, thus increasing the likelihood that they will receive help when they need it.

\section{Why Do Debt-Dased Transfers Continue to Exist?}

This leads to the question of why need-based and debtbased transfers coexist as they do among the Maasai and in many other societies around the world. We propose that each one is appropriate in specific but quite different 
circumstances. When needs arise on a regular, predictable basis, it is possible for people to agree to a balanced exchange of favors: You help me today, I'll help you tomorrow. When such favors are not repaid, it also makes sense to end the relationship and try again with someone else. In these debt-based relationships, the possibility that the relationship will end because of a lack of repayment makes individuals wary about engaging in debt-based interactions with valued partners. It also may be hard to enforce repayment in this kind of situation. For example, Samburu do not engage in esile transactions with affines, brothers, or isotuatin: "you could not argue with them if they don't repay you" (Perlov, 1987:185). But when needs arise unpredictably, it is sensible to simply help those in need so that they will still be around to help you if you yourself are in need at some point in the future.

This is also well illustrated by a pattern of sharing called 'neighboring' that we have observed among ranchers in the American Southwest (Cronk, 2015; Cronk et al., 2019, Cronk et al., 2021). Neighboring has two varieties, one that corresponds with debt-based transfers and another that corresponds with need-based transfers. For help with predictable chores, such as rounding up livestock for branding or marketing, ranchers agree to trade favors in the form of skilled labor, and most ranchers expect such shared labor to be repaid. But when needs arise unpredictably (e.g., due to injuries, illnesses, and deaths), most ranchers provide help to those in need with no expectation of any repayment beyond a similar kindness should they themselves ever suffer from a similarly unpredictable need.

\section{Network Sizes and Pastoralist Survival}

In our models, a major determinant of agents' survival was simply the sizes of the networks they were in: agents who happened to be in large networks survived, on average, at higher rates than agents in smaller networks. This may have implications for the survival of pastoralists and pastoralism in the real world, as well. If pastoralist communities are shrinking due to, for example, outmigration and the adoption of other subsistence modes, this could reduce the viability of their risk-pooling networks and threaten the survival of such communities even further. However, this effect might be counterbalanced, at least in part, if support from other economic activities, such as wage labor, enabled them to manage the risks of pastoralism in other ways such as improving veterinary care, buying commercial insurance (e.g., Bertram-Huemmer \& Kraehnert, 2018; Takahashi et al., 2018), or simply saving money in anticipation of hard times.
Need-Based Transfers may be Relevant for Disaster Response and Recovery

Our findings may have implications for real-world disaster response. When disasters occur asynchronously in the model, agents survive longer if they use a need-based transfer strategy rather than a debt-based strategy and if networks are larger and more modular. Because it may be difficult in many circumstances to simply create larger or more modular networks, perhaps the most important take-home message is that, when disasters and other negative events arise unpredictably, it may be more adaptive to simply give to those in one's network who are in need if one is able to do so and to do so without any expectation of repayment, rather than to give with the understanding that the relationship will end if the gift is not repaid. Such spontaneous helping networks often do emerge in the aftermath of disasters, with individuals helping strangers without expecting anything in return (Ripley, 2009; Solnit, 2010). For example, after the 1906 earthquake in San Francisco, even traditionally marketdriven exchanges, such as food deliveries and transportation, became need-based, with food being distributed and public transportation running for free until after the crisis passed (Solnit, 2010). These examples, combined with the modelling results we report above, suggest that need-based helping systems may be an important part of the human toolkit for surviving disasters.

\section{Glossary}

Social Network

Analysis (SNA)

Regular Network 


\section{Small World Network}

\section{Preferential \\ Attachment Network}

(i.e., number of connections with other nodes).

A small world network is a network where the typical distance, L, between two randomly chosen nodes (the number of steps required) grows proportionally to the logarithm of the number of nodes $\mathrm{N}$ in the network (Watts \& Strogatz, 1998). At the same time, the clustering coefficient is not small. In the context of a social network, this results in the small world phenomenon of strangers being linked by a short chain of acquaintances. Many empirical graphs show the small world effect, e.g., social networks, the underlying architecture of the Internet, wikis such as Wikipedia, and gene networks.

Preferential attachment is the mechanism used in generating the networks. Intuitively, preferential attachment can be understood if we think in terms of social networks connecting people. Here a link from $\mathrm{A}$ to $\mathrm{B}$ means that person A "knows" a person B. Heavily linked nodes represent well-known people with lots of relations. When newcomers enter a community, they are more likely to become acquainted with one of those more visible people rather than with a relative unknown. The Barabási-Albert model (Barabási \& Albert, 1999) is an algorithm for generating modular random scalefree networks using a preferential attachment mechanism. Several natural and human made systems, including the Internet, citation networks, and some social networks are thought to be approximately scale-free and certainly contain few nodes (called hubs) with unusually high degree as compared to the other nodes of the network.
Degree

The degree of a node in a network is the number of connections or edges that connect the node to other nodes (see Freeman, 1978). An edge is defined as a line segment that connects two nodes.

Modularity Modularity is one measure of the structure of networks or graphs. It is designed to measure the strength of division of a network into modules (also called groups, clusters or communities; Newman, 2006). Networks with high modularity have dense connections between the nodes within modules but sparse connections between nodes in different modules.

Supplementary Information The online version contains supplementary material available at https://doi.org/10.1007/s10745-021-00273-6.

Acknowledgements This material is based upon work supported by the John Templeton Foundation. Any opinions, findings, conclusions, or recommendations expressed in this material are those of the authors and do not necessarily reflect the views of the John Templeton Foundation.

Author Contribution MC, LC, and AA conceived of the study, designed the study, coordinated the study and helped draft the manuscript. MC created the original model, accomplished the simulations and carried out the statistical analyses. All authors gave final approval for publication.

Funding This material is based upon work supported by a grant from the John Templeton Foundation.

Data Availability The model is publicly available in the Computational Model Library of CoMSES (https://www.comses.net/codebases/).

\section{Declarations}

Ethics Approval This section does not apply.

Competing Interests The authors declare no competing financial interests.

Open Access This article is licensed under a Creative Commons Attribution 4.0 International License, which permits use, sharing, adaptation, distribution and reproduction in any medium or format, as long as you give appropriate credit to the original author(s) and the source, provide a link to the Creative Commons licence, and indicate if changes were made. The images or other third party material in this article are included in the article's Creative Commons licence, unless indicated otherwise in a credit line to the material. If material is not included in the article's Creative Commons licence and your intended use is not permitted by statutory regulation or exceeds the permitted use, you will need to obtain permission directly from the copyright holder. To view a copy of this licence, visit http://creativecommons.org/licenses/by/4.0/. 


\section{References}

Aktipis, A., de Aguiar, R., Flaherty, A., Iyer, P., Sonkoi, D., \& Cronk, L. (2016). Cooperation in an uncertain world: For the Maasai of East Africa, need-based transfers outperform account-keeping in volatile environments. Human Ecology, 44(3), 353-364. https:// doi.org/10.1007/s10745-016-9823-z

Aktipis, C. A., Cronk, L., \& de Aguiar, R. (2011). Risk-Pooling and Herd Survival: An Agent-Based Model of a Maasai Gift-Giving System. Human Ecology, 39(2), 131-140.

Almagor, U. (1978). Pastoral Partners: Affinity and Bond Partnership Among the Dassanetch of South-West Ethiopia. Manchester Univ. Press.

Ash, J., \& Newth, D. (2007). Optimizing complex networks for resilience against cascading failure. Physica A: Statistical Mechanics and Its Applications, 380, 673-683.

Barabàsi, A. L., \& Albert, R. (1999). Emergence of scaling in random networks. Science, 286, 509-512.

Bertram-Huemmer, V., \& Kraehnert, K. (2018). Does Index Insurance Help Households Recover from Disaster? Evidence from IBLI Mongolia. American Journal of Agricultural Economics, 100, 145-171.

Bettencourt, L. M., Lobo, J., Helbing, D., Kühnert, C., \& West, G. B. (2007). Growth, innovation, scaling, and the pace of life in cities. PNAS, 104(17), 7301-7306.

Bird, R. B., Bird, D. W., Smith, E. A., \& Kushnick, G. C. (2002). Risk and reciprocity in Meriam food sharing. Evolution and Human Behavior, 23(4), 297-321.

Bollig, M. (1998). Moral economy and self-interest: Kinship, friendship, and exchange among the Pokot (NW Kenya). In Schweizer, T., and White, D. (eds.), Kinship, Networks, and Exchange. Cambridge University Press, Cambridge. Cambridge University Press, Cambridge, pp. 137-157.

Bollig, M. (2006). Risk Management in a Hazardous Environment: A Comparative Study of Two Pastoral Societies (Vol. 2). Springer.

Bullmore, E., \& Sporns, O. (2012). The economy of brain network organization. Nature Reviews Neuroscience, 13(5), 336-349.

Cashdan, E. A. (1985). Coping with Risk: Reciprocity Among the Basarwa of Northern Botswana. Man, 20(3), 454-474.

Cronk, L. (2007). The Influence of Cultural Framing on Play in the Trust Game: A Maasai Example. Evolution and Human Behavior, 28(5), 352-358.

Cronk, L. (2015). "Neighboring": a preliminary look at generosity and mutual aid among ranchers in the American Southwest (http://humangenerosity.org).

Cronk, L., Aktipis, A. (2021) Design principles for risk-pooling systems. Nature Human Behaviour 5, 825-833.

Cronk, L., Berbesque, C., Conte, T., Gervais, M., Iyer, P., McCarthy, B., \& Aktipis, A. (2019). Managing risk through cooperation: Need-based transfers and risk pooling among the societies of the Human Generosity Project. In Global Perspectives on LongTerm Community Resource Management, Ludomir R. Lozny and Thomas H. McGovern, eds. Springer.

Cronk, L., Guevara Beltran, D., Mercado, D., \& Aktipis, A. (2021). "A solidarity-type world": Need-based helping among ranchers in the southwestern United States. Human Nature, 32 (2), 482-508.

Dahl, G., \& Hjort, A. (1976). Having Herds: Pastoral Herd Growth and Household Economy. University of Stockholm, Stockholm.

Dixit, A. K., Levin, S. A., \& Rubenstein, D. I. (2013). Reciprocal insurance among Kenyan pastoralists. Theoretical Ecology, 6(2), 173-187.

Dyson-Hudson, N. (1966). Karimojong Politics. Clarendon.

Eriksen, K. A., Simonsen, I., Maslov, S., \& Sneppen, K. (2003). Modularity and extreme edges of the Internet. Physical review letters, 90(14), 148701.
Fafchamps, M. (2011). Risk Sharing Between Households. In Handbook of Social Economics, Jess Benhabib, Alberto Bisin, and Matthew O. Jackson (eds.). San Diego and Amsterdam: North-Holland.

Fafchamps, M., \& Lund, S. (2003). Risk-Sharing Networks in Rural Philippines. Journal of Development Economics, 71, 261-287.

Flannery, K., Marcus, J., \& Reynolds, R. (1989). The Flocks of the Wamani: A Study of Llama herders on the Punas of Ayacucho, Peru. Academic, New York.

Freeman, L. C. (1978). Centrality in social networks conceptual clarification. Social Networks, 1(3), 215-239.

Garas, A., Argyrakis, P., \& Havlin, S. (2008). The structural role of weak and strong links in a financial market network. The European Physical Journal B-Condensed Matter and Complex Systems, 63(2), 265-271.

Guimera, R., Mossa, S., Turtschi, A., \& Amaral, L. N. (2005). The worldwide air transportation network: Anomalous centrality, community structure, and cities' global roles. PNAS, 102(22), 7794-7799.

Gulliver, P. (1955). The Family Herds: A Study of Two Pastoral Tribes in East Africa: the Jie and Turkana. International Library of Sociology and Social Reconstruction.

Hao, Y., Armbruster, D., Cronk, L., \& Aktipis, A. (2015a). Needbased transfers on a network: A model of risk-pooling in ecologically volatile environments. Evolution and Human Behavior. https://doi.org/10.1016/j.evolhumbehav.2014.12.003

Hao, Y., Armbruster, D., \& Hütt, M. T. (2015b). Node survival in networks under correlated attacks. PloS One, 10(5):e0125467.

Hollander, M., \& Wolfe, D. A. (1973). Nonparametric Statistical Methods. John Wiley \& Sons.

Iyer, P. (2016). Risk Management Strategies of Male and Female Pastoralists in Karamoja, Uganda. Ph.D. dissertation, anthropology, Rutgers University.

Lenzu, S., \& Tedeschi, G. (2012). Systemic risk on different interbank network topologies. Physica A: Statistical Mechanics and Its Applications, 18(391), 4331-4341.

Mol, F. (1996). Maasai Language and Culture Dictionary. Lemek: Maasai Centre.

Moritz, M. (2016). Networks and hazard adaptation among West African pastoralists. In Social Network Analysis of Disaster Response, Recovery, and Adaptation. Eric C. Jones and A. J. Fass, eds., pp. 193-207. Elsevier.

Moritz, M., Giblin, J., Ciccone, M., Davis, A., Fuhrman, J., Kimiaie, M., Madzsar, S., Olson, K., \& Senn, M. (2011). Social risk-management strategies in pastoral systems: A qualitative comparative analysis. Cross-Cultural Research, 45(3), 286-317.

Newman, M. E. (2006). Modularity and community structure in networks. PNAS, 103(23), 8577-8582.

Perlov, D. C. (1987). Trading for influence: The social and cultural economics of livestock marketing among the highland Samburu of Northern Kenya. Ph.D. dissertation, anthropology, University of California - Los Angeles.

Potkanski, T. (2000). Mutual assistance among the Ngorongoro Maasai. In The Poor Are Not Us: Poverty and Pastoralism in Eastern Africa. David M. Anderson and Vigdis Broch-Due, eds., pp. 199-217. James Currey Ltd.

Ripley, A. (2009). The unthinkable: Who survives when disaster strikes - and why. Harmony.

Smith, D., Dyble, M., Major, K., Page, A. E., Chaudhary, N., Salali, G. D., Thompson, J., Vinicius, L., Migliano, A. B., \& Mace, R. (2019). A friend in need is a friend indeed: Need-based sharing, rather than cooperative assortment, predicts experimental resource transfers among Agta hunter-gatherers. Evolution and Human Behavior, 40(1), 82-89.

Solnit, R. (2010). A paradise built in hell: The extraordinary communities that arise in disaster. Penguin. 
Steinbacher, M., Steinbacher, M., \& Steinbacher, M. (2016). Robustness of banking networks to idiosyncratic and systemic shocks: A network-based approach. Journal of Economic Interaction and Coordination, 11, 95. https://doi.org/10.1007/s11403-014-0143-3

Takahashi, K., Barrett, C. B., \& Ikegami, M. (2018). Does Index Insurance Crowd In or Crowd Out Informal Risk Sharing? Evidence from Rural Ethiopia. American Journal of Agricultural Economics, 101, 672-691.

Townsend, C., Aktipis, A., Balliet, D., \& Cronk, L. (2020). Generosity among the Ik of Uganda. Evolutionary Human Sciences 2, e23.

Turnbull, C. (1972). The Mountain People. Simon \& Schuster.

Waller, R. (1988). Emutai: crisis and response in Maasailand 18831902. In The Ecology of Survival. Case Studies from Northeast
African History (Eds D. Johnson and D. Anderson). Pp. 73-114. Lester Crook Academic Publishing, London.

Watts, D. J., \& Strogatz, S. H. (1998). Collective dynamics of 'smallworld' networks. Nature, 393, 440-442.

Wiessner, P. (1982). Risk, reciprocity and social influences on! Kung San economics. In E. Leacock \& R. Lee (Eds.), Politics and history in (Vol. 61, pp. 61-84). Cambridge University Press.

Wilensky, U. (1999). NetLogo. http://ccl.northwestern.edu/netlogo/. Center for Connected Learning and Computer-Based Modeling, Northwestern University, Evanston, IL.

Publisher's Note Springer Nature remains neutral with regard to jurisdictional claims in published maps and institutional affiliations. 